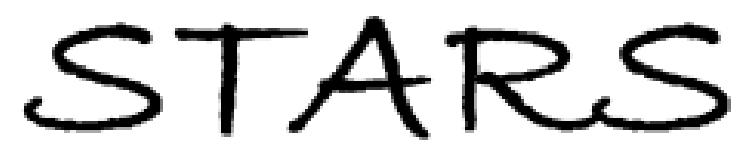

University of Central Florida

STARS

$1-1-2003$

\title{
LIM kinase 1 is essential for the invasive growth of prostate epithelial cells - Implications in prostate cancer
}

\author{
Monica Davila \\ University of Central Florida \\ Andra R. Frost \\ William E. Grizzle \\ Ratna Chakrabarti \\ University of Central Florida
}

Find similar works at: https://stars.library.ucf.edu/facultybib2000

University of Central Florida Libraries http://library.ucf.edu

This Article is brought to you for free and open access by the Faculty Bibliography at STARS. It has been accepted for inclusion in Faculty Bibliography 2000s by an authorized administrator of STARS. For more information, please contactSTARS@ucf.edu.

\section{Recommended Citation}

Davila, Monica; Frost, Andra R.; Grizzle, William E.; and Chakrabarti, Ratna, "LIM kinase 1 is essential for the invasive growth of prostate epithelial cells - Implications in prostate cancer" (2003). Faculty Bibliography 2000s. 2561.

https://stars.library.ucf.edu/facultybib2000/2561

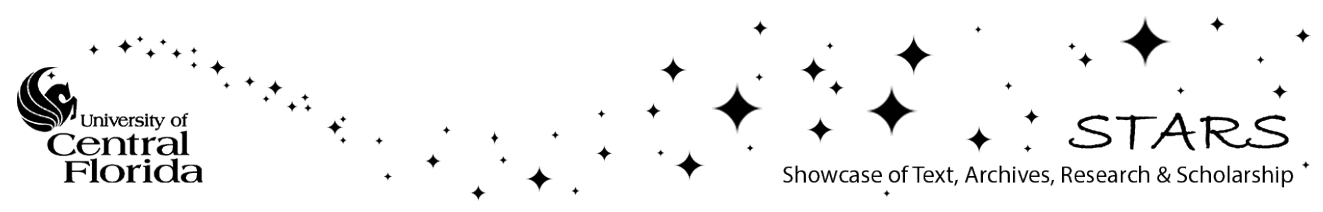




\title{
LIM Kinase 1 Is Essential for the Invasive Growth of Prostate Epithelial Cells
}

\author{
IMPLICATIONS IN PROSTATE CANCER*
}

Received for publication, June 12, 2003

Published, JBC Papers in Press, June 23, 2003, DOI 10.1074/jbc.M306196200

\author{
Monica Davila $\ddagger$ Andra R. Frost\$, William E. Grizzle§, and Ratna Chakrabartiłף \\ From the $\ddagger$ Department of Molecular Biology and Microbiology University of Central Florida, Orlando, Florida 32826 \\ and §Department of Pathology, University of Alabama, Birmingham, Alabama 35294
}

\begin{abstract}
Mammalian LIM kinase 1 (LIMK1) is involved in reorganization of actin cytoskeleton through inactivating phosphorylation of the ADF family protein cofilin, which depolymerizes actin filaments. Maintenance of the actin dynamics in an ordered fashion is essential for stabilization of cell shape or promotion of cell motility depending on the cell type. These are the two key phenomena that may become altered during acquisition of the metastatic phenotype by cancer cells. Here we show that LIMK1 is overexpressed in prostate tumors and in prostate cancer cell lines, that the concentration of phosphorylated cofilin is higher in metastatic prostate cancer cells, and that a partial reduction of LIMK1 altered cell proliferation by arresting cells at $G_{2} / M$, changed cell shape, and abolished the invasiveness of metastatic prostate cancer cells. We also show that the ectopic expression of LIMK1 promotes acquisition of invasive phenotype by the benign prostate epithelial cells. Our data provide evidence of a novel role of LIMK1 in regulating cell division and invasive property of prostate cancer cells and indicate that the effect is not mediated by phosphorylation of cofilin. Our study correlates with the recent observations showing a metastasisassociated chromosomal gain on $7 q 11.2$ in prostate cancer, suggesting a possible gain in LIMK1 DNA (7q11.23).
\end{abstract}

LIM kinase 1 (LIMK1 $)^{1}$ belongs to a novel dual specificity (serine/threonine and tyrosine) kinase family that contain two amino-terminal LIM domains (1). LIMK1 gene is expressed predominantly in brain and in developing neural tissues (2), and its deletion (microdeletion of chromosome 7q11.23) is typical for Williams syndrome (3). Cofilin, one of the actin-binding proteins, considered to be a potent regulator of the actin dynamics (4) by means of its activity in F-actin depolymerization, is the only known substrate of LIMK1. The function of cofilin is inhibited by phosphorylation at the Ser-3 residue (5) by LIMK1, which leads to accumulation of F-actin. The catalytic

\footnotetext{
* The costs of publication of this article were defrayed in part by the payment of page charges. This article must therefore be hereby marked "advertisement" in accordance with 18 U.S.C. Section 1734 solely to indicate this fact.

I Supported by NCI, National Institutes of Health Grant CA81329 and by an award from the Florida Hospital Gala Endowment for Oncologic Research. To whom correspondence should be addressed: Dept. of Molecular Biology and Microbiology, University of Central Florida, 12722 Research Pkwy., Orlando, FL 32826. Tel.: 407-882-2258; Fax: 407-384-2062; E-mail: rchak@mail.ucf.edu.

1 The abbreviations used are: LIMK1, LIM kinase 1; NGF, nerve growth factor; EGF, epidermal growth factor; $\mathrm{BPH}-1$, benign prostatic hyperplasia cells.
}

activity of LIMK1 is regulated by distinct members of the Rho subfamily of small GTPases (Rho, Rac, and Cdc42), which controls actin filament dynamics and focal adhesions assembly in response to extra- and intracellular stimuli. Rho, Rac, and Cdc42 induce formation of stress fibers, assembly of lamellipodia and membrane ruffles, and regulation of filopodial protrusions, respectively (6). LIMK1 has been shown to mediate specifically Rac-induced actin cytoskeleton reorganization and focal adhesion complexes $(5,7,8)$. Rac-induced activation of LIMK1 is mediated by PAK1, which phosphorylates LIMK1 on its $\mathrm{Thr}^{508}$ residue (9). Other studies also proposed that Rhoand Cdc42-induced cytoskeletal changes are mediated through phosphorylation of LIMK1 by Rho-dependent protein kinase ROCK (10) and Cdc42-regulated protein kinase PAK4 (11) and $\operatorname{MRCK} \alpha(12)$.

The non-catalytic domain of LIMK1 contains two tandem repeats of a LIM motif, a putative zinc binding motif, and a PDZ domain, which contains two tandem nuclear exit signal sequences (7). LIMK1 also contains a nuclear localization signal-like basic cluster sequence. The non-catalytic domain of LIMK1 has been shown to have effects on cytoskeleton reorganization independent of its kinase activity. Specific regions of the non-catalytic domain (LIM or PDZ) inhibit neurite outgrowth in differentiating PC12 cells in response to Ras, neuronal growth factor (NGF) or a ROCK inhibitor without altering endogenous LIMK1 activity $(13,14)$. These observations suggest that the non-catalytic domain of LIMK1 is involved in regulating cellular processes though protein-protein interaction. LIMK1 also physically interacts through its LIM domain with the cytoplasmic tail of neuregulins, a family of transmembrane proteins that functions as receptor tyrosine kinase ligands. These proteins are known to be involved in the regulation of synapse formation and maintenance, cell proliferation, apoptosis, differentiation, and neuronal migration (15). Deletion experiments have shown that the lack of LIM and PDZ domains enhances the effect of LIMK1 on actin cytoskeleton (16). In addition, expression of a kinase-inactive form of the protein blocked the effect of the native LIMK1 (5). These findings suggest an inhibitory role of the non-catalytic domain in the regulation of the kinase activity of LIMK1.

Reorganization of cytoskeleton is an essential feature of motility, detachment, and invasion of cancer cells. Individual members of the Rho family, such as Rac and Cdc42, induce distinct actin remodeling events, work together at the leading edge of the cell, and coordinate lamellipodial and filopodial extensions, whereas Rho activates accumulation of stress fibers through activation of ROCK (17). Formation and stabilization of actin filaments are the key events downstream of Rac and Cdc42, which provide the protrusive force for cellular extensions at the leading edge of the migratory cells (18). Seemingly, 
these events are critical for the invasive behavior of the cancer cells, as the inhibition of Rho kinase activity has been shown to reduce invasive progress of prostate cancer cells in vivo (19). Rho proteins are activated by growth factor receptors and their ligands, for example, EGF and NGF and their respective receptors erbB2 and Trk. Cellular-erbB2 and Trk are often overexpressed and activated in various types of cancers including prostate cancer (20-22). Furthermore, c-erbB2 was shown to enhance the invasiveness and metastatic potential of cancer cells (20). Similarly, NGF and Trk expressions in prostate cancer cells coincide with transformation to a malignant phenotype capable of invading along the perineural space and extracapsular metastasis to a distant site (23). As evident from membrane ruffling and lamellipodia formation, EGF- and NGF-induced increased invasion of prostate tumor cells is mediated through Rac (24). Therefore, it is likely that Rac-mediated activation of actin reorganization is mediated through LIMK1. cDNA microarry and differential display reverse-transcription PCR analyses revealed an up-regulation of LIMK1 mRNA (25) and LIMK1 as the elastin-linked gene (26) in prostate adenocarcinoma cells. This study reports that LIMK1 is overexpressed in prostate adnocarcinomatous tissues and in malignant prostate cell lines and is essential for the invasive property and growth of prostate cancer cells, and the effect is not mediated through inactivating phosphorylation of cofilin.

\section{EXPERIMENTAL PROCEDURES}

Cell Culture, Transfection, and Isolation of Stable Cell Line-PC3, DU145, LNCaP (ATCC), PrEC (Clonetics), BPH-1 (a gift from P. Narayan, Celebration, FL), p69, M21, and M12 (gift from J. Ware, Richmond, VA) were maintained in RPMI (DU145, LNCaP, p69, M21, and M12), Ham-12 (PC3), DMEM (BPH-1) and in specified medium (PrEC) and grown to log phase before being used as described (27). PC3 cells were transfected using LipofectAMINE (Invitrogen) with the open reading frame of the LacZ gene or the antisense cDNA of the coding sequence of LIMK1 (1.94 kilobases), both cloned into an ecdysoneinducible mammalian expression vector (pIND, Invitrogen). BPH-1 cells (benign prostatic hyperplasia) were transfected with the open reading frames of LIMK1 or LacZ cloned in pIND. Cells were cotransfected with the vector $\mathrm{pVRxR}$ that encodes the subunit of a heterodimer of the ecdysone and the retinoid $\mathrm{X}$ receptor to establish stable cell lines (PC3ASL and PC3LacZ) capable of expressing antisense LIMK1 RNA or LacZ when induced with an ecdysone analogue, ponasterone A. Double stable clones were selected using $500 \mu \mathrm{g} / \mathrm{ml}$ Geneticin (Invitrogen) and $50 \mu \mathrm{g} / \mathrm{ml}$ Zeocin (Invitrogen). When mentioned, stably transfected cells were continuously induced during the times indicated by adding $5 \mu \mathrm{M}$ ponasterone A (Invitrogen) every $22 \mathrm{~h}$.

Immunoblotting, Immunohistochemistry, and ImmunofluorescenceTotal cell lysates were resolved in SDS-PAGE (10-14\%) and subjected to immunoblotting using anti-LIMK1 (Transduction Laboratories), anti-cofilin (Cytoskeleton), or anti-phospho-cofilin antibodies (Cell Signaling). Anti-glyceraldehyde-3-phosphate dehydrogenase antibody (Biogenesis) was simultaneously used as an internal control. Goat antimouse or anti-rabbit antibody was used as the secondary antibody. Paraffin-embedded tissue sections of normal and malignant human prostate were subjected to a pepsin-based antigen retrieval protocol (BioGenex, San Ramon, CA) followed by incubation with anti-LIMK1 antibody. A biotinylated multi-link goat anti-immunoglobulin for mouse, rabbit, guinea pig, and rat was used as the secondary antibody. Positive signals were detected by horseradish peroxidase-conjugated streptavidin and 3,3'-diaminobenzidine (DAB) as the chromogen (BioGenex Multi-Link kit). For indirect immunofluorescence, PC3ASL and PC3LacZ cells were plated on poly-L-lysine-coated glass cover slips and continuously induced for $72 \mathrm{~h}$. Cells were permeabilized and dually stained with Alexa Fluor 488-conjugated phalloidin (Molecular Probes) and anti-LIMK1, anti-paxillin (Transduction Laboratories), or antiMT1-MMP (Cytoskeleton) antibodies. Cy3-conjugated anti-mouse or anti-rabbit antibody (Molecular Probes) was used as the secondary antibody. Fluorescent images were captured in a Zeiss LSM 510 laserscanning confocal microscope.

In Vivo Phosphorylation, Expression of Recombinant Protein, and Immunecomplex Kinase Assay-BPH-1 and PC3 cells were cultured in the presence of $1.5 \mathrm{mCi}$ of $\left[{ }^{32} \mathrm{P}\right]$ orthophosphate for $10 \mathrm{~h}$. Equal amounts of total cell lysates were subjected to immunoprecipitation with anti- a

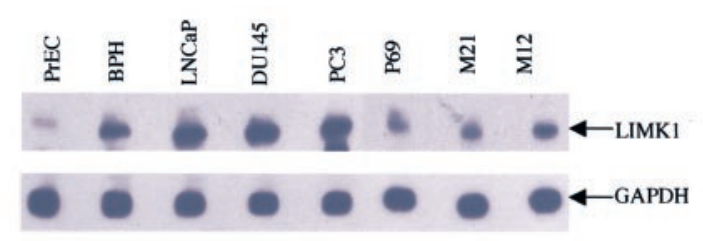

b
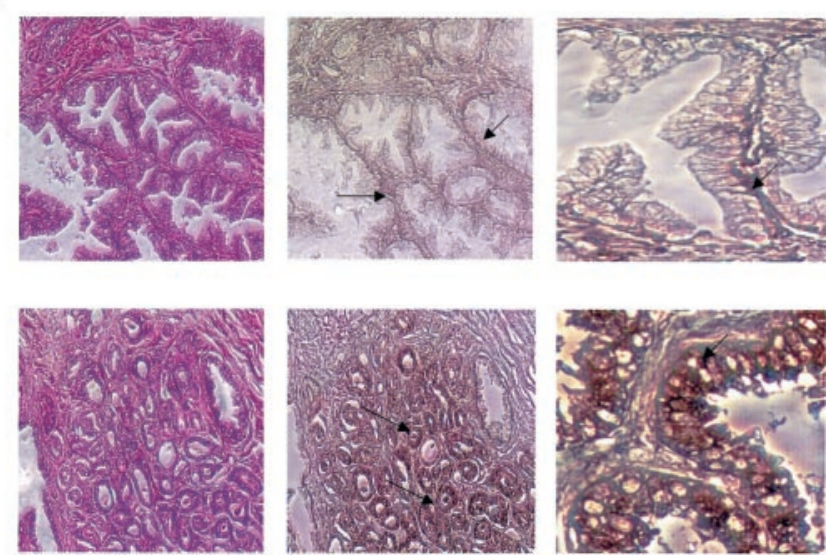

FIG. 1. LIMK1 is differentially expressed in human prostate cell lines and prostate tissues. $a$, immunoblot analysis of LIMK1 in total lysates of human prostate cell lines. $b$, expression of LIMK1 in prostate tissues. Upper panel, Normal/benign areas. Lower panel, cancerous glands. Upper and lower left, Hematoxylin/eosin-stained slides. Magnification, $\times 100$. Upper and lower right, areas from normal and cancerous tissues are shown in higher magnification $(\times 200)$. Arrows indicate light staining in basal cells in normal/benign areas (upper panel) and intense staining in cells in cancerous areas (lower panel). $\operatorname{PrEC}$, prostate epithelial cells. GAPDH, glyceraldehyde-3-phosphate dehydrogenase.

cofilin antibody and protein A-agarose beads (Sigma) using the procedure as described (28), and the products were resolved in SDS-PAGE (14\%). Labeled cofilin was detected by autoradiography and PhosphorImager analysis (Amersham Biosciences). Recombinant cofilin was expressed in Escherichia coli as a His-tagged fusion protein by cloning the open reading frame of human cofilin into the $\mathrm{T} 7$ polymerase driven pET30Ek/LIC (Novagen) expression vector using specific primers (F, 5'-GACGACGACAAGATGGCCTCCGGTGTGGCTG-3', and R, 5' -GAGGAGAAGCCC GGTTCACAAAGGCTTGCCC-3'). The expression construct was used to transform BL21 (DE3) E. coli cells. Transformed cells were induced with $1 \mathrm{~mm}$ isopropyl-1-thio- $\beta$-D-galactopyranoside at $25^{\circ} \mathrm{C}$, and the expressed cofilin was purified through a Nickel affinity column (HiTrap Chelating HP columns, Amersham Biosciences).

For immune complex kinase assay, LIMK1 was immunoprecipitated from PC3 cells cultured under regular conditions using anti-LIMK1 antibody and protein G-agarose (Sigma) beads. Protein G-agarose beads bound to the immune complex were resuspended in kinase assay buffer (50 mM HEPES, pH 7.5, $150 \mathrm{~mm} \mathrm{NaCl}, 50 \mu \mathrm{M}$ ATP, $5 \mathrm{~mm} \mathrm{MgCl}_{2}, 5 \mathrm{~mm}$ $\left.\mathrm{MnCl}_{2}, 10 \mathrm{~mm} \mathrm{NaF}, 1 \mathrm{~mm} \mathrm{Na} \mathrm{VO}_{4}, 3 \mathrm{nM}\left[\gamma_{-}{ }^{32} \mathrm{P}\right] \mathrm{ATP}\right)$ and incubated at $30{ }^{\circ} \mathrm{C}$ for $20 \mathrm{~min}$ with $10 \mu \mathrm{g}$ of recombinant cofilin as the substrate (29). The reaction mix was resolved in SDS-PAGE (14\%), and phosphorylated cofilin was detected by autoradiography and PhosphorImager analysis.

Invasion Assay-Cells were plated in serum-free media (containing ponasterone A for transfected cells) on control and Matrigel-coated membrane containing invasion chambers (BD Biosciences). Cells were incubated at $37^{\circ} \mathrm{C}$ for $22 \mathrm{~h}$ in a $\mathrm{CO}_{2}$ incubator using $5 \%$ fetal bovine serum in the lower chamber as the chemoattractant. Invading cells were stained with Diff-Quick ${ }^{\mathrm{TM}}$ stain (DADE) and counted. The percentage of cells that invaded through the Matrigel-coated membranes was calculated by comparison with the cells passed through the membranes in the control chambers.

$\left[{ }^{3} H\right]$ Thymidine Incorporation, Cell Synchronization, and Fluorescence-activated Cell Sorter Analysis-Asynchronous PC3LacZ and PCASL cells were incubated with $1 \mu \mathrm{Ci}$ of $\left[{ }^{3} \mathrm{H}\right]$ thymidine in the presence or absence of ponasterone A. At different time points after induction, cells were treated with $5 \%$ trichloroacetic acid and lysed using 0.5 $\mathrm{N} \mathrm{NaOH}$. Incorporation of $\left[{ }^{3} \mathrm{H}\right]$ thymidine to DNA was measured in a scintillation counter (Beckman LS 5000 TD). For cell cycle analysis, 

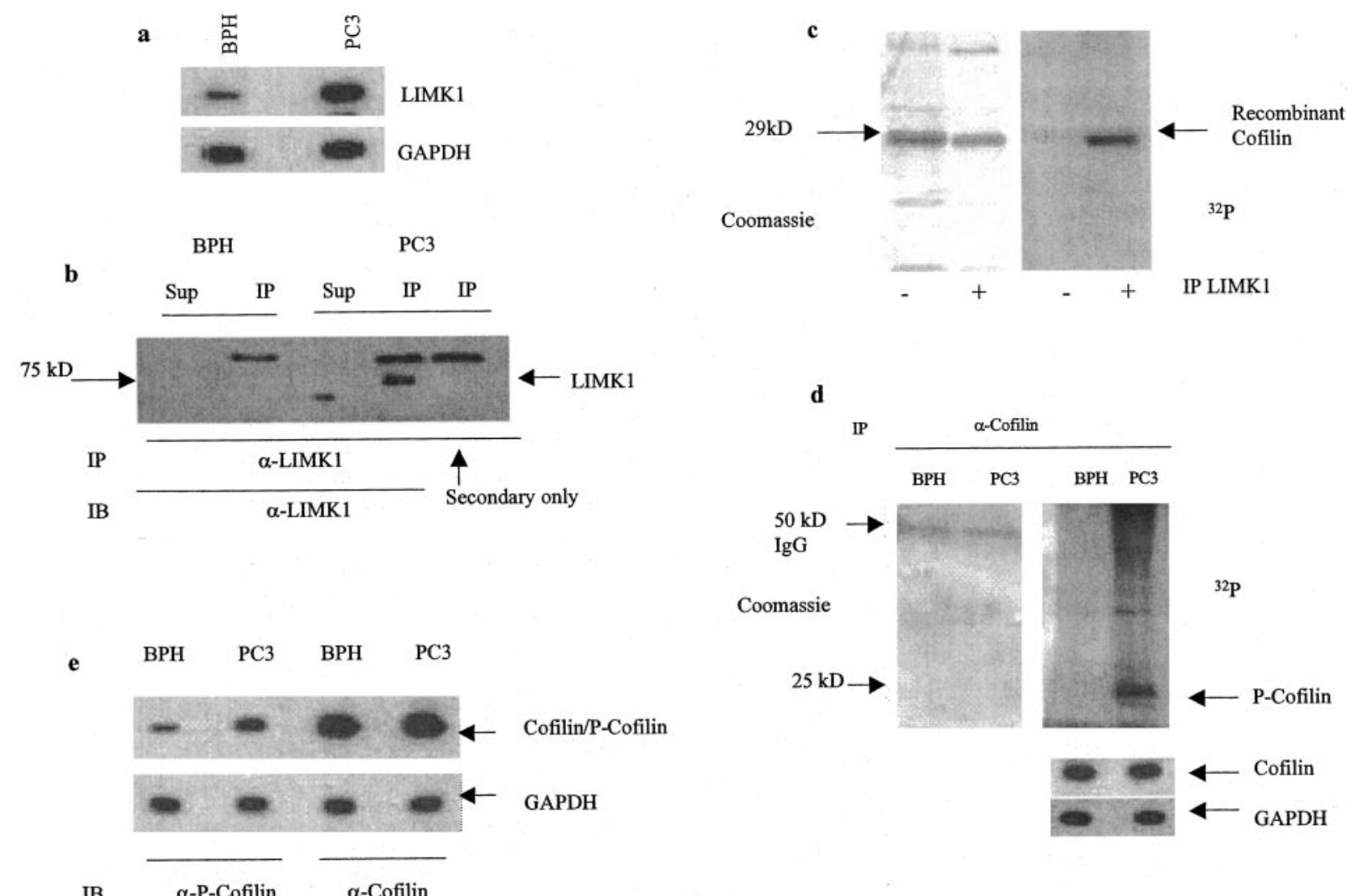

FIG. 2. A higher concentration of phosphorylated cofilin is associated with overexpression of LIMK1 in PC3 cells. $a$ and $b$, LIMK1 concentration in BPH-1 and PC3 cells before $(a)$ and after $(b)$ immunoprecipitation (IP) with anti-LIMK1 antibody. IB, immunoblot. Sup, supernatant. $c$, in vitro phosphorylation of recombinant cofilin by LIMK1 immunoprecipitated from PC3 cells. $d$, in vivo phosphorylation of cofilin in BPH-1 and PC3 cells. Immunoblot analysis of cell lysates before immunoprecipitation shows the initial concentration of cofilin in both samples (lower panel). e, immunoblot analysis of phosphorylated cofilin or cofilin in crude cell extracts using anti-phospho-cofilin or anti-cofilin antibodies. $G A P D H$, glyceraldehyde-3-phosphate dehydrogenase.

PC3LacZ and PC3ASL cells induced every $22 \mathrm{~h}$ were synchronized at the $\mathrm{G}_{1} / \mathrm{S}$ boundary by a double thymidine block by treating them with thymidine $(2 \mathrm{mM})$ for $24 \mathrm{~h}$ followed by an 8 -h release in fresh growth medium and successive retreatment with thymidine $(2 \mathrm{~mm})$ for $16 \mathrm{~h}$. Cells were then released to enter the cell cycle in fresh growth medium and harvested at specified times. Cells were fixed in paraformaldehyde (1\%) in phosphate-buffered saline (PBS), washed in PBS, permeabilized with saponin $(0.25 \%)$ in PBS, and treated with RNase $(1 \mathrm{mg} / \mathrm{ml})$ at room temperature. Cells were washed in phosphate-buffered saline and stained with propidium iodide $(400 \mu \mathrm{g} / \mathrm{ml})$ at $37^{\circ} \mathrm{C}$ for $30 \mathrm{~min}$. Cells were analyzed in a flow cytometer (FACScalibur BD Biosciences), and raw data were analyzed using Modfit (BD Biosciences) software.

\section{RESULTS}

LIMK1 Is Overexpressed in Cancerous Prostate Cells and Tissues and Is Catalytically Active-To confirm the differential expression of LIMK1 in prostate cancer, we monitored the expression profile of LIMK1 in various prostate cell lines and prostate tissues. Our results revealed an increased expression of LIMK1 in highly aggressive metastatic prostate epithelial cells and in some prostatic adenocarcinomas (Fig. 1). The concentration of LIMK1 in some cancerous prostate cells (M21 and M12) increased proportionately with increasing tumorigenic and aggressive properties compared with normal prostate epithelial cells (P69) and BPH-1 cells (Fig. 1a). A 2-3-fold higher expression of LIMK1 was noted in tumorigenic (LNCaP and M21) and metastatic (PC3, DU145, and M12) prostate cancer cells compared with BPH-1 and P69 (non-tumorigenic) cells (Fig. 1a). Compared with prostate epithelial cells, the expression of LIMK1 was 5-10-fold higher in the metastatic prostate cancer cells (PC3 and M21) (Fig. 1a). Consistent with these findings, immunohistochemical analysis indicated a highly abundant expression of LIMK1 in some cancerous glands in prostatic epithelium (Fig 1b). The expression of LIMK1 in prostate tissues was localized to the basal cells in benign glands (arrows in Fig. 1b, top: middle and right) compared with a widespread staining in both the cytoplasm and the nucleus in the epithelial cells in cancerous glands (arrows in Fig. 1b, bottom: middle, and right). These results suggest a possible correlation between overexpression of LIMK1 and phenotypic differences in prostate tissues.

The abundantly expressed LIMK1 in prostate cancer cells was catalytically competent as immunoprecipitated LIMK1 (Fig. 2, $a$ and $b$ ) from PC3 cells was capable of phosphorylating recombinant cofilin (Fig. 2c), the only known substrate of LIMK1 (5). To assess whether the increased expression of LIMK1 was associated with an increased phosphorylation of endogenous cofilin, the phosphorylation status of the native cofilin in BPH-1 and PC3 cells was studied using in vivo phosphorylation and immunoblotting techniques. Although no significant difference in the expression of cofilin was noted in these two cell lines an appreciable increase in the phosphorylated cofilin was noted in PC3 cells (Fig. 2, $d$ and $e$ ), which demonstrates a parallel increase in phosphorylated cofilin in these cells.

A Partial Inhibition of LIMK1 Expression Did Not Result in Reduced Phosphorylation of Cofilin-To understand the possible role of overexpressed LIMK1 in acquisition of tumorigenic and metastatic phenotypes, we used antisense-RNA mediated functional inactivation of LIMK1; subsequently, we generated a stable cell line (PC3ASL) capable of expressing antisense LIMK1 RNA after induction with an ecdysone analogue, ponasterone A. A time course study using PC3ASL and a control transformed cell line, PC3LacZ, after continuous induction ev- 

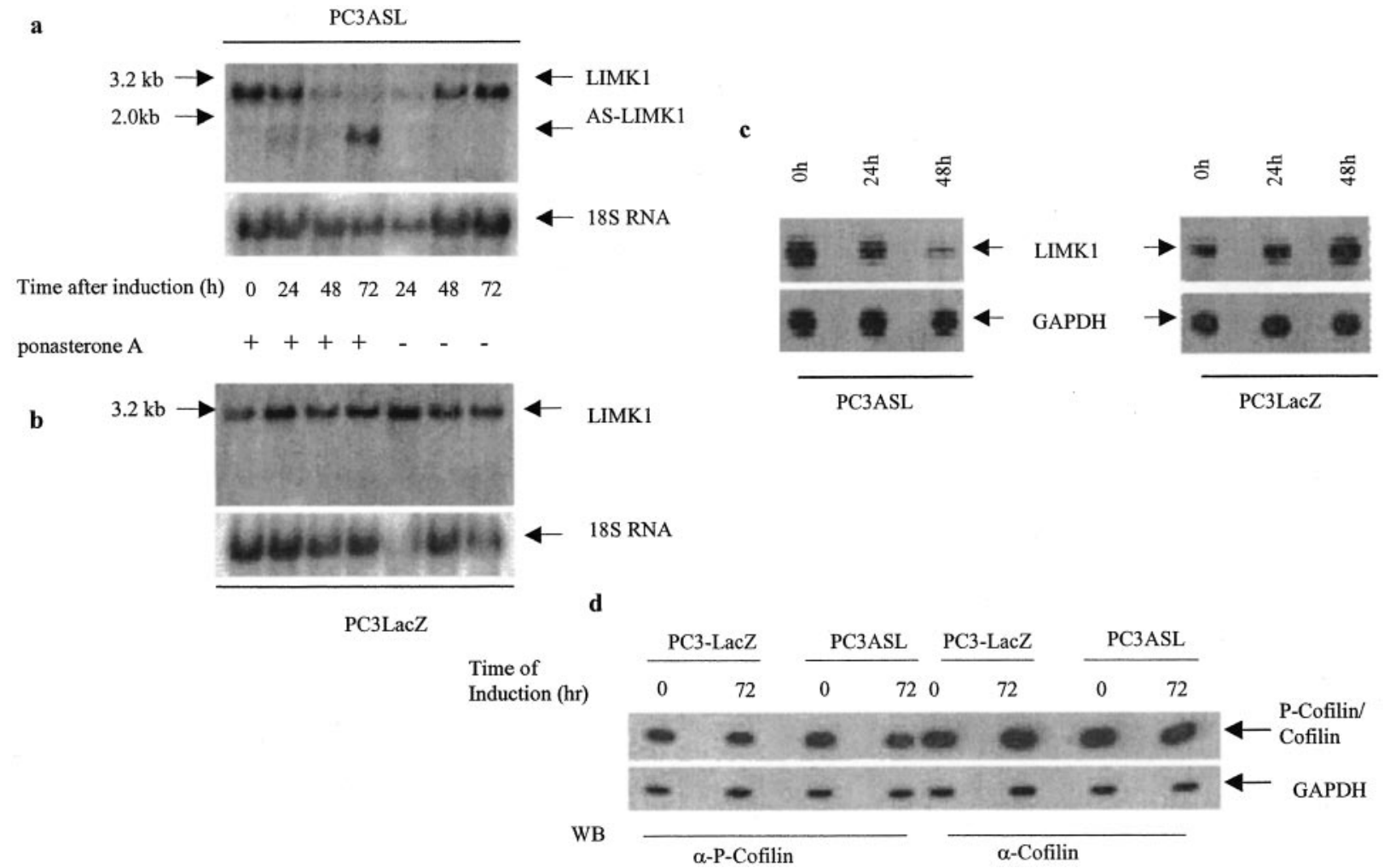

FIG. 3. LIMK1 was effectively reduced in PC3ASL cells by expression of antisense LIMK1, but there was no concurrent reduction in cofilin phosphorylation. $a$ and $b$, RNA blot analysis of LIMK1 in PC3ASL $(a)$ and PC3LacZ (b) cells induced successively (+) or not ( - ). AS, antisense. $c$, concentration of LIMK1 in transfected PC3 cells harvested at different time points after successive induction. $d$, concentration of phosphorylated cofilin (P-cofilin) or cofilin in transfected PC3 cells after continuous induction. Despite the reduced concentration of LIMK1 no noticeable difference in phospho-cofilin concentration was detected in PC3ASL cells. WB, Western blot. GAPDH, glyceraldehyde-3-phosphate dehydrogenase.

ery $20-22 \mathrm{~h}$ for $72 \mathrm{~h}$ indicated the maximum expression of LIMK1 antisense transcript at $72 \mathrm{~h}$, which was associated with a concurrent decrease in the concentration of the sense LIMK1 transcript only in the induced PC3ASL cells (Fig. 3a). No decrease in the LIMK1 transcript was apparent in any of the PC3LacZ cells induced or not or in uninduced PC3ASL cells (Fig. 3, $a$ and $b$ ). A parallel reduction in the LIMK1 protein concentration confirmed the decreased synthesis of LIMK1 in PC3ASL cells (Fig. 3c). Immunoblot analysis using anti-cofilin or anti-phospho-cofilin antibodies revealed no simultaneous decrease in the concentration of phosphorylated cofilin in PC3ASL cells (Fig. 3d). This observation suggests two possibilities, either the residual LIMK1 can be catalytically more efficient and maintain an increased concentration of phosphorylated cofilin or the enhanced phosphorylation of cofilin was not mediated by LIMK1. Although cofilin is the only known substrate of LIMK1, it can be phosphorylated by other serine/ threonine kinases, such as LIMK2 (8) and TESK1/TESK2 (30).

Reduced Expression of LIMK1 Retarded Cell Proliferation by Arresting Cells at $G_{2} / M$ Phase-The effect of reduced expression of LIMK1 on cell proliferation and cell cycle progression was studied next. Because LIMK1 is expressed in a growth phase-dependent manner, which suggests its possible involvement in regulation of cell growth (31), we monitored the growth pattern of induced and non-induced PC3ASL and PC3LacZ cells by $\left[{ }^{3} \mathrm{H}\right]$ thymidine incorporation assay. Our results indicated that the inhibition of expression of LIMK1 led to a block in $\left[{ }^{3} \mathrm{H}\right]$ thymidine incorporation only in PC3ASL cells after $48 \mathrm{~h}$ of continuous induction (Fig. 4a), signifying a growth arrest in PC3ASL cells. A possible role of active LIMK1 in regulating mitosis has been suggested also, based on the studies that have shown that the LIMK1 activity fluctuates with cell cycle pro- gression and attains a maximum level during mitosis when LIMK becomes hyperphosphorylated, presumably by mitotic Cdks $(29,32)$. We used induced PC3ASL and PC3LacZ cells synchronized at $\mathrm{G}_{1} / \mathrm{S}$ boundary by double thymidine block and monitored cell cycle progression by harvesting cells at different time points after the release of the block. At $0 \mathrm{~h}, \sim 75-78 \%$ of PC3ASL and PC3LacZ cells were at $\mathrm{G}_{1} / \mathrm{S}$ phase. The remaining $23-25 \%$ of cells were at $\mathrm{G}_{2} / \mathrm{M}$ phase, which could be accounted for by the higher DNA content in a subpopulation of PC3 cells with inherent chromosomal gain (33). After the release of thymidine block, both cell types exhibited progression through $\mathrm{S}$ phase ( $\sim 60 \%$ cells $)$ and $\mathrm{G}_{2} / \mathrm{M}(\sim 30 \%$ cells $)$ during the next $6 \mathrm{~h}$. PC3LacZ cells started to cycle through $\mathrm{G}_{1}$ as a gradual increase in the percent of cells in $G_{1}$ was noted with a concurrent decrease in the total percent of cells in $S$ and $G_{2} / M$ phases between 6-14 h (Fig. 4, $b$ and $c$ ). In contrast, PC3ASL cells started to accumulate in $\mathrm{G}_{2} / \mathrm{M}$ phase during this time $(\sim 50 \%$ cells at $14 \mathrm{~h}$ ), indicating a $\mathrm{G}_{2} / \mathrm{M}$ arrest in these cells (Fig. $4, b$ and $d$ ). No block in the cell cycle progression in PC3LacZ cells (Fig. 4, $b$ and $c$ ) confirms that a threshold concentration of LIMK1 is necessary for the passage of cells through $\mathrm{G}_{2} / \mathrm{M}$ phase. This result is also consistent with the $\left[{ }^{3} \mathrm{H}\right]$ thymidine incorporation data, indicating a growth arrest in PC3ASL cells.

Reduced Expression of LIMK1 Abolished the Invasive Behavior of Prostate Cancer Cells-The effect of inhibition of LIMK1 expression on the invasive property of PC3 cells was studied next using an in vitro invasion assay. Consistent with their highly metastatic ability, a 3-fold higher percentage of untransfected PC3 cells were invasive and migrated through the Matrigel compared with the non-invasive BPH-1 cells (Fig. 5, a and $b$ ). A partial inhibition of LIMK1 expression in PC3ASL cells resulted in a 4-5-fold decrease in the percent of invaded 
a

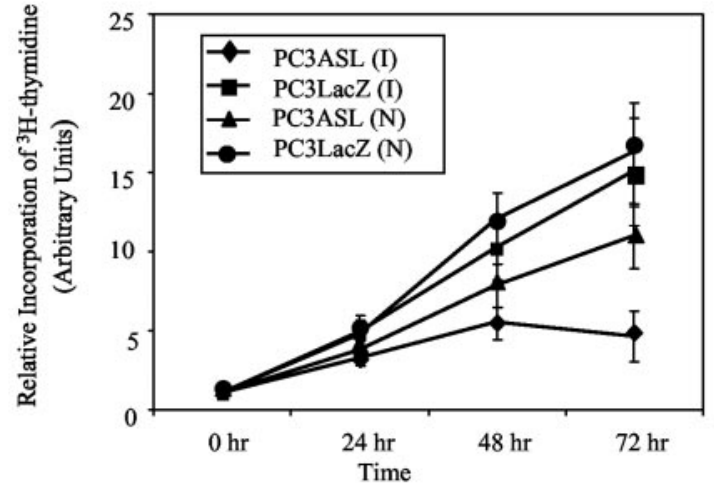

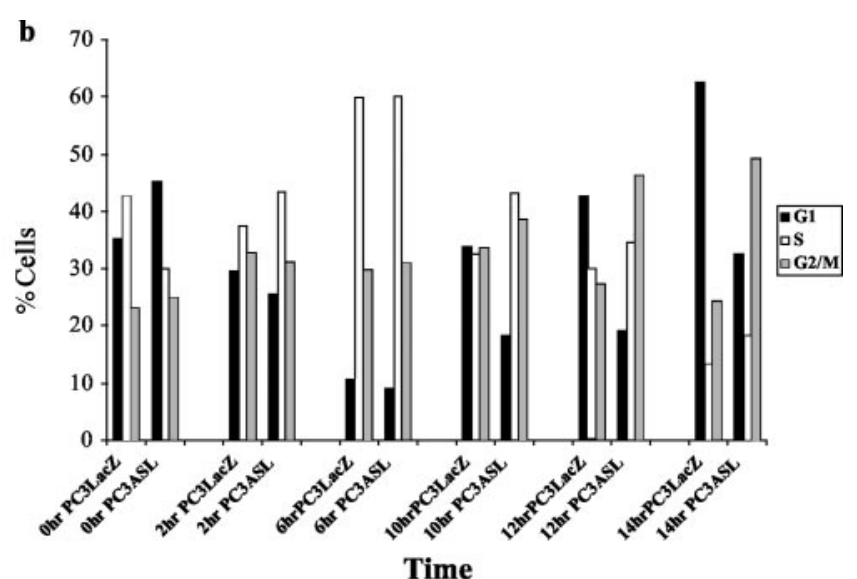

c
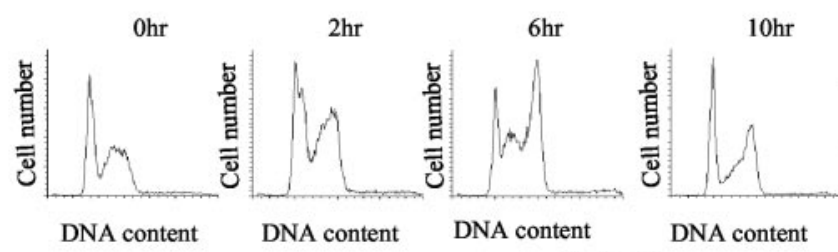
PC3LacZ
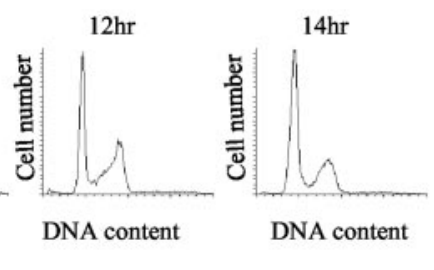

d
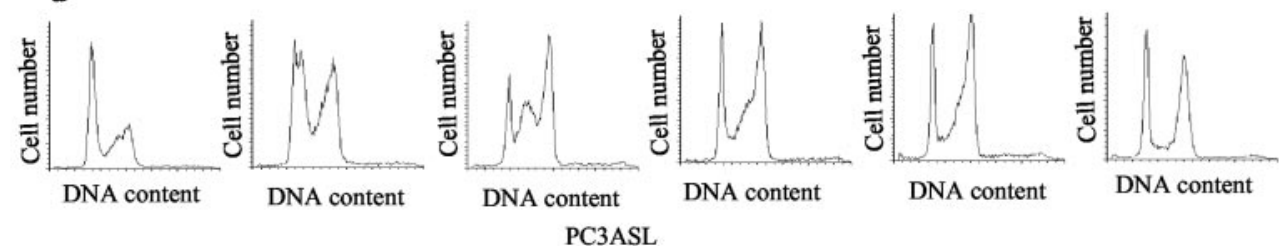

FIG. 4. The reduced expression of LIMK1 in PC3 cells is associated with suppression of cell growth and cell cycle arrest in $\mathrm{G}_{2} / \mathrm{MI}$ phase. $a,\left[{ }^{3} \mathrm{H}\right]$ thymidine incorporation in PC3ASL and PC3LacZ cells induced successively $(I)$ or not $(N)$. Data represent values relative to the initial time point $(0 \mathrm{~h})$ and as the mean \pm S.E of three independent experiments. $b-d$, flow cytometric analysis of cell cycle transition in synchronized PC3LacZ $(c)$ and PC3ASL $(d)$ cells successively induced for $72 \mathrm{~h}$. Cells were arrested at the $\mathrm{G}_{1} / \mathrm{S}$ boundary and released. $b$, percentage of cells at different stages of cell cycle. Cell cycle profiles show similar progression of PC3ASL and PC3LacZ cells to the $\mathrm{S}$ and $\mathrm{G}_{2} / \mathrm{M}^{\mathrm{p}}$ phase up to $6 \mathrm{~h}$ after release, but only PC3LacZ cells continue to $\mathrm{G}_{1}$ and $\mathrm{S}$ phase, whereas PC3AL cells undergo a $\mathrm{G}_{2} / \mathrm{M}$ arrest from $10 \mathrm{~h}$ onwards.

cells compared with that in the wild type PC3 and PC3LacZ cells (transfection control), which was comparable with the percent of BPH-1 cells invaded through the membranes. These data confirm that the functional LIMK1 is essential for the invasive function of PC3 cells.

To further confirm the role of LIMK1 in promoting invasiveness of prostate epithelial cells, the effect of ectopic expression of LIMK1 or LacZ (as the control) in non-invasive BPH-1 cells was studied. Expression of LIMK1 in transfected BPH-1 cells after induction by ponasterone A showed predominantly cytoplasmic localization of LIMK1 (Fig. $5 d$ ) and altered the growth pattern from a clustered and patchy appearance (BPHLacZ)) to a more uniform distribution of single cells (BPHL) (Fig. 6, $i$ and j). More importantly, expression of LIMK1 in ponasterone Atreated BPHL cells significantly increased the percentage of invasive cells (13-fold) compared with BPH-1 cells expressing LacZ (Fig. 5, $a$ and $c$ ), suggesting acquisition of a new invasive phenotype by the non-invasive prostate cells. This study provides compelling evidence that LIMK1 is one of the key proteins that promotes invasiveness and suggests that the increased expression of LIMK1 in PC3 cells may render them metastatic.

Altered Expression of LIMK1 Changes Cell Morphology and Organization of Actin Cytoskeleton-The dynamic regulation of actin cytoskeleton and the adhesion apparatus and the extracellular matrix degradation are both required for the invasion process of tumor cells. Therefore, we monitored possible alterations in these processes following reduced expression of
LIMK1 in PC3 cells. The induced PC3LacZ cells exhibited cell morphology that is typically observed in PC3 cells, with smooth edges and the occasional presence of the filopodial spike-like structures extended from one or both ends of the cell and, in some cases, a phase dense border, characteristic of ruffling lamellipodia around an extensive area of the cell periphery (Fig. 6a, arrows). A majority of induced PC3ASL cells, however, showed a more flattened and irregular shape, with several dark patches at the periphery of the cell (arrows in Fig. 6b) that appear to be possible adhesion points. Fluorescent staining of actin cytoskeleton showed a prominent actin meshwork, characteristic of lamellipodia formation at the leading edge of the induced PC3LacZ cells (Fig. 6c, middle and right); this meshwork was also typical of parental PC3 cells (data not shown) and of motile cells. Immunolocalization of LIMK1 (Fig. 6c) showed a perinuclear distribution, with distinct targeting to the ruffles at the leading edge of these cells (arrows in Fig. $6 c$, left). In contrast, a high percentage of induced PC3ASL cells showed a predominant formation of discrete stress fibers and focal adhesions typically observed in non-motile adherent cells (34) (Fig. 6d, middle and right). Distribution of LIMK1 in these cells was also perinuclear, with one or more broad areas of accumulation (arrows) at the periphery of the cell (Fig. 6d, left).

Any change in the adhesion system in PC3ASL and PC3LacZ cells was investigated next by monitoring immunolocalization of paxillin, an adapter protein associated with focal adhesions of adherent cells $(17,35,36)$ (Fig. 6e). Similar to the parental PC3 cells (data not shown), PC3LacZ cells showed the presence 


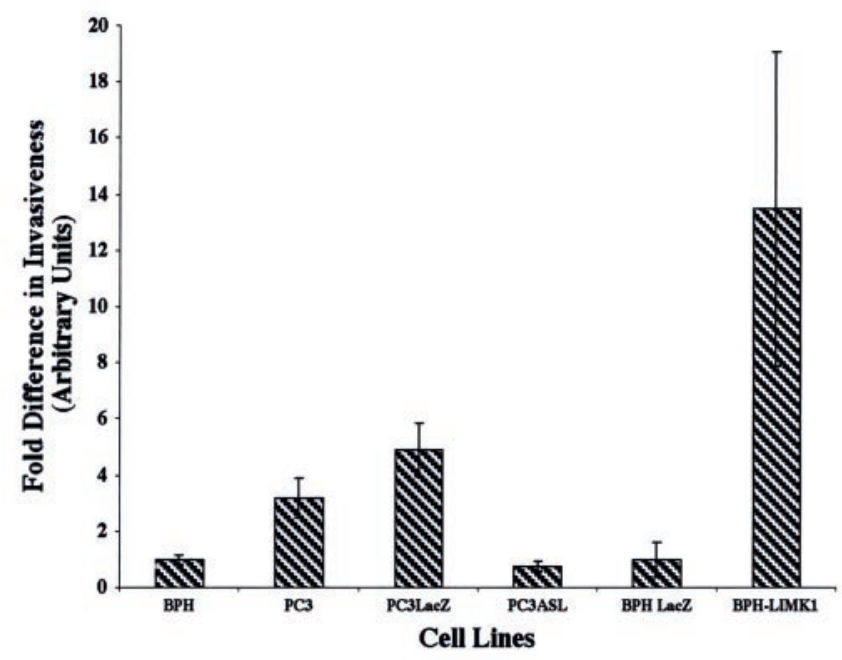

d

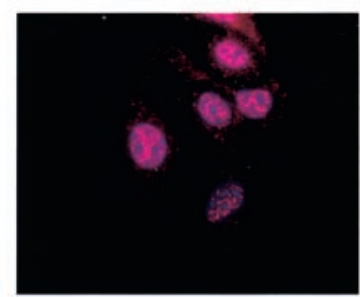

BPHLacZ

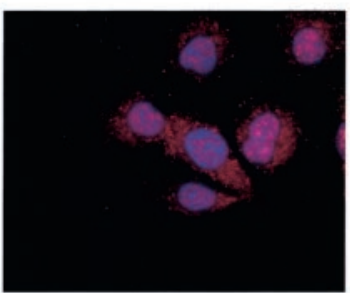

BPHL

b

BPH

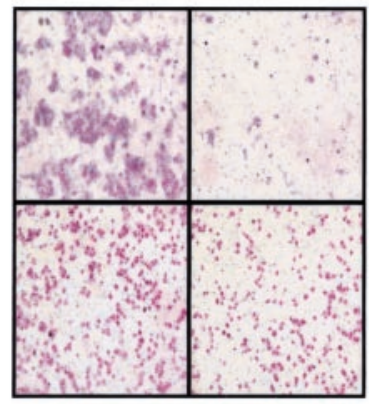

Control

Matrigel

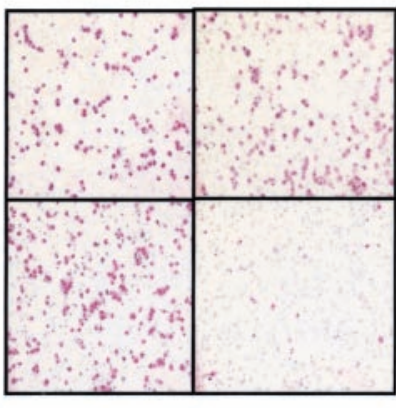

BPH1

Matrigel

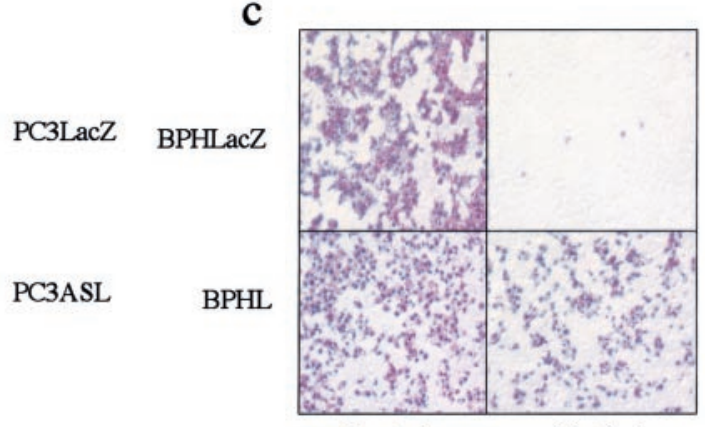

Control

FIG. 5. Altered expression of LIMK1 is associated with changes in the invasiveness of prostate cells. Reduced expression of LIMK1 is associated with a reversion of the invasion ability of PC3 cells. Transfected cells were successively induced for $48 \mathrm{~h}$ before being plated in to the invasion chambers. Ectopic expression of LIMK1 in BPH-1 cells increased invasion significantly after induction with ponasterone A for 24 h. $a$, fold difference in invasion in different cell lines. Data represents mean \pm S.E. of quantitative analysis of three independent assays (each from two different clones of transfected PC3 and BPH-1 cells). $b$ and $c$, bright field images of cells invaded to the underside of the control and Matrigel membranes. $d$, staining of LIMK1 in BPHL and BPHLacZ cells showing predominantly cytoplasmic distribution of LIMK1 in BPHL cells. Nuclei were stained with 4,6-diamidino-2-phenylindole $(\times 200)$.

of paxillin as broad areas surrounding the nucleus and small adhesion sites at the cell periphery, which overlapped the actin meshwork that forms the lamellipodia (arrows in Fig. 6e, left, middle, and right). In contrast, paxillin was more centrally localized in PC3ASL cells, with frequent accumulation in broad areas between the actin meshwork (arrows) representative of stress fibers (Fig. 6f, left, middle, and right) and the cell boundary.

Published reports indicated an elevated expression of the membrane-type matrix metalloproteinase MT1-MMP in invasive prostate cancer cells (38) such as PC3 and DU145 and its intense staining in selected secretory glands in cancerous prostate tissues (39). Because the localization of MT1-MMP to the lamellipodia has been correlated with the invasive properties of cells $(40-42)$ we monitored the distribution of this protein in PC3ASL and PC3LacZ cells. Distribution of MT1-MMP in induced PC3LacZ was localized to the perinuclear and to the lamellipodia along with actin (arrows in Fig. 6g, left, middle, and right) but mainly in the perinuclear region in PC3ASL cells
(Fig. 6h) and between actin stress fibers (enlarged box). These results suggest that a reduction in LIMK1 concentration induced changes in the actin cytoskeleton reorganization, the adhesion pattern, and localization of MT1-MMP in PC3 cells.

\section{DISCUSSION}

In this paper we have shown that the expression of LIMK1, one of the proteins that modulates actin dynamics, is up-regulated in adenocarcinomatous prostatic epithelium and prostate cancer cell lines. The degree of expression of LIMK1 showed a correlation with the aggressiveness of cancer cells. For example, expression of LIMK1 was higher in metastatic PC3 cells and M12 cells compared with less-aggressive LNCaP and M21 cells. Consistent with this observation, expression of LIMK1 was significantly high in cancerous prostate tissues compared with histologically normal prostatic epithelium. This finding may explain the consequence of the chromosomal gain on $7 q 11.2-q 31$ that is predominantly found in distant metastasis of prostate tumors $(43,44)$. Molecular cytogenetic analysis of 
$\mathbf{a}$

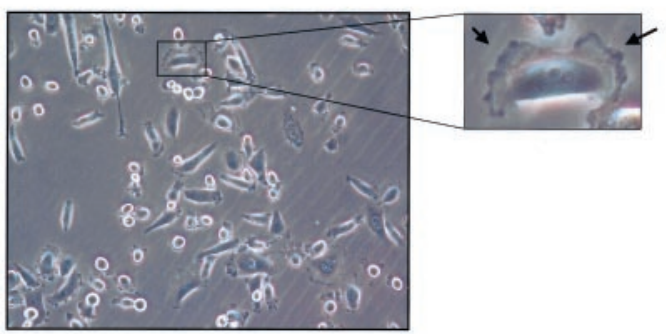

b

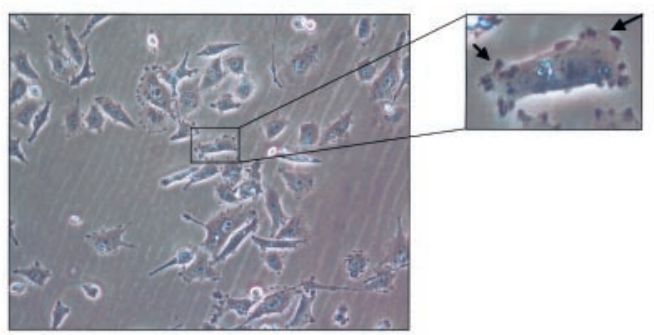

i

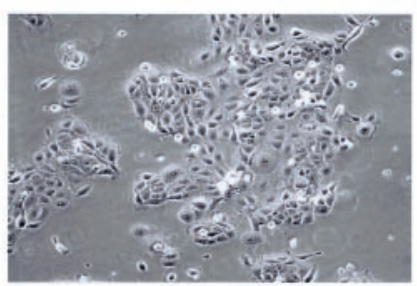

j

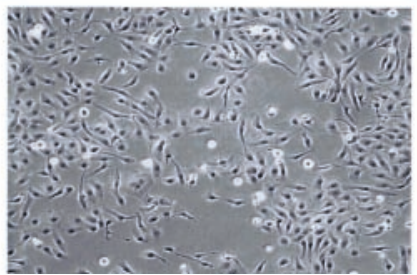

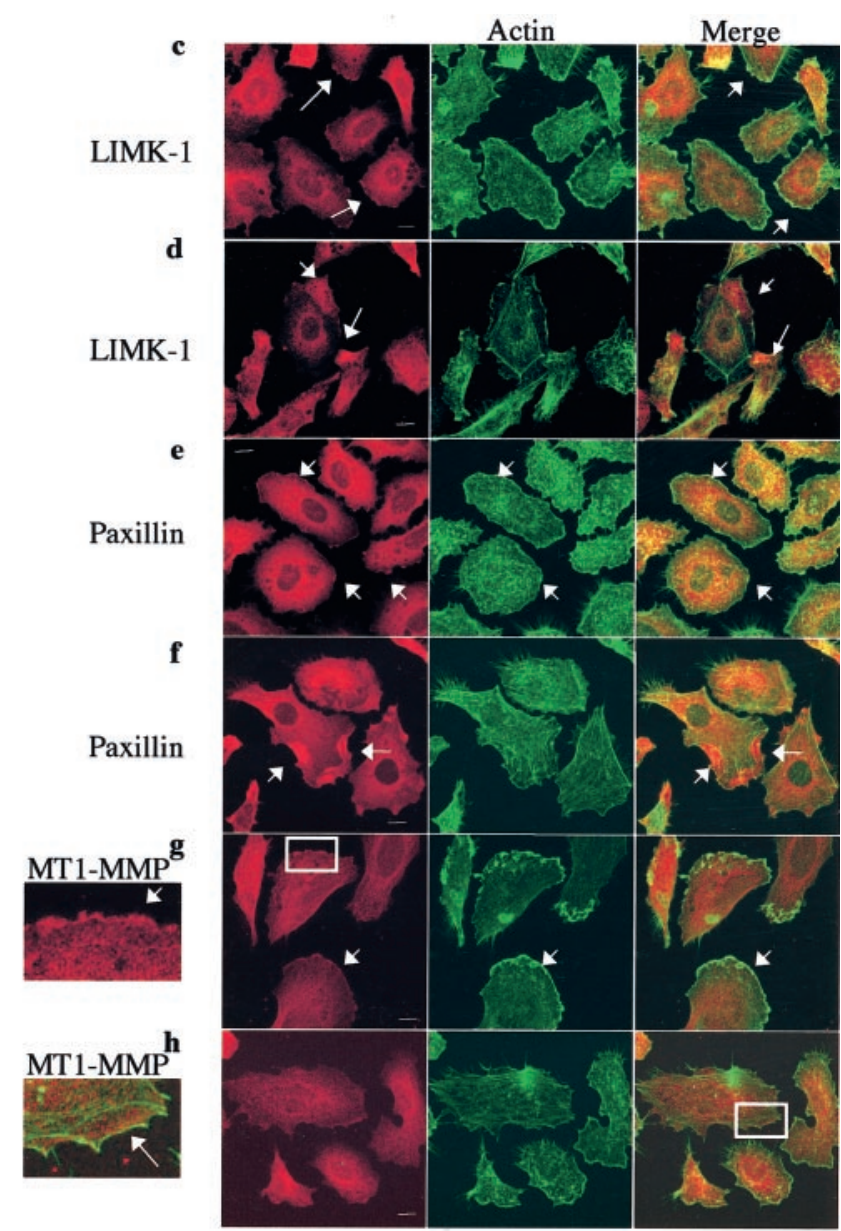

FIG. 6. Reduced expression of LIMK1 is associated with changes in cell morphology, cytoskeleton organization, and the invasive ability of PC3 cells. $a$ and $b$, phase-contrast images of PC3LacZ $(a)$ and PC3ASL (b) cells after successive induction for $72 \mathrm{~h} . c-h$, dual staining of continuously induced PC3LacZ $(c, e$, and $g$ ) and PC3ASL $(d, f$, and $h$ ) cells with Alexa 488-conjugated phalloidin and anti-LIMK1 ( $c$ and $d$ ), anti-paxillin $(e$ and $f)$, or MT1-MMP $(g$ and $h$ ) antibodies. Arrows indicate localization of LIMK1 in lamellipodia along with actin $(c)$, accumulation of LIMK1 between actin stress fibers and cell boundary $(d)$, localization of paxillin to the small adhesion points at the lamellipodia along with actin $(e)$, redistribution of paxillin and accumulation in large areas between actin stress fibers and cell periphery $(f)$, and localization of MT1-MMP (also in the enlarged section) and actin to the lamellipodia ( $g$ ). Scale bar, $10 \mu \mathrm{m} . i$ and $j$, phase contrast images of BPHLacZ $(i)$ and BPHL $(j)$ showing the difference in growth pattern.

prostatic adenocarcinomas indicated a gradual increase for the number of patients with gain of chromosome $7 \mathrm{pq}$ with increasing tumor volume (45). Importantly, these chromosomal alterations are also found in primary tumors that showed progression after radical prostatectomy (46), suggesting a possible correlation of LIMK1 expression as a biomarker for prostate cancer progression.

To understand the implication of up-regulated LIMK1 in prostate cancer, we used BPH-1 and PC3 cells as models for benign and cancerous prostate cells. PC3 cells are widely used for studies on invasive behavior of prostate cancer, which also exhibited increased expression of LIMK1 compared with $\mathrm{BPH}-1$. Because the function of LIMK1 is mediated through phosphorylation of cofilin in reorganization of actin cytoskeleton, we studied the status of the kinase activity of immunoprecipitated LIMK1 from PC3 cells, which confirmed that the endogenous LIMK1 was catalytically active. Interestingly, a parallel increase in the concentration of endogenous phosphocofilin was also noted in PC3 cells. However, no significant decrease in the concentration of phospho-cofilin was seen after enforced reduction of the expression of LIMK1. Presumably, in PC3 cells, phosphorylation of cofilin is not solely mediated by LIMK1. A number of studies show that protein kinases differ- ent from LIMK1 phosphorylate cofilin at Ser-3 as well as at multiple other sites $(8,28,47)$. Although unlikely, it is also possible that the residual LIMK1 in PC3ASL cells is highly efficient catalytically and can maintain a higher concentration of phospho-cofilin.

Our study on antisense-RNA-mediated reduced expression of LIMK1 provides evidence on the involvement of LIMK1 in regulation of growth of prostate cancer cells, specifically at the $\mathrm{G}_{2} / \mathrm{M}$ phase of the cell cycle. Recent studies show that LIMK1 undergoes mitosis-specific activation by hyperphosphorylation, which is associated with a concomitant increase in phosphorylation of cofilin $(29,32)$. LIMK1 becomes activated in prometaphase and metaphase and comes back to the basal level as cells enter telophase (32), which suggests that a controlled activity of cofilin by phosphorylation and dephosphorylation is necessary for mitosis. Our study indicates a direct role of LIMK1 in $\mathrm{G}_{2} / \mathrm{M}$ phase, as a partial reduction of LIMK1 expression induced a $\mathrm{G}_{2} / \mathrm{M}$ arrest in PC3ASL cells. However, it is uncertain whether the effect of the reduced concentration of LIMK1 was mediated through the increased activity of cofilin due to nonphosphorylation since no alteration in phospho-cofilin concentration was noted in these cells. It is possible that either a subpopulation of dephosphorylated cofilin was main- 
tained in the nucleus that could not be phosphorylated in PC3ASL cells upon reduced expression of LIMK1 or the role of LIMK1 is mediated through its interaction with other proteins. Further studies are needed to dissect the process of LIMK1mediated regulation of mitosis.

A direct role of LIMK1 in promoting cellular invasion, a hallmark of metastasis, is also evident from our studies on Matrigel-based invasion assay. A partial inhibition of LIMK1 reduced the percentage of invading cells to a level comparable with the BPH-1 cells. However, the effect of ectopic expression of LIMK1 in BPH-1 cells on promotion of invasion was more pronounced and significantly higher than the highly metastatic PC3 cells, suggesting a possible role LIMK1 in the acquisition of metastatic behavior of prostate tumors. Accordingly, we have noted a significantly increased expression of LIMK1 in metastatic prostate tumors (data not shown), indicating an association of LIMK1 expression with advanced prostate cancer. A possible involvement of the Rac, an upstream effector of LIMK1, in NGF- and EGF-induced increased invasion of prostate cancer has been proposed (24). It is possible that the effect of growth factors on the increased invasion is mediated through LIMK1 in advanced prostate cancer in which specific receptors for NGF and EGF are overexpressed $(21,22)$. However, it is unclear how the ectopic expression of LIMK1 promotes cellular invasion in BPH-1 cells, in which the upstream effectors of LIMK1 may not be activated in the absence of specific extracellular signals.

Consistent with the effect of LIMK1 in promotion of cellular invasiveness, a visible alteration in the cell shape and adhesion pattern was caused by a partial reduction in LIMK1 in prostate epithelial cells. PC3ASL cells showed discrete stress fibers, and a more flattened appearance that was typically observed after Rho activation (17). Interestingly, the effect of LIMK1 in facilitating cell invasion is not mediated through the inactivating phosphorylation of cofilin. This observation suggests a presently unknown area of possible interactions between LIMK1 and other proteins that are involved in maintenance of cell behavior such as invasion and locomotion. The presence of two LIM domains and a PDZ domain at the amino-terminal end of LIMK1 $(1,37)$ suggests its possible interaction with other proteins. It is speculated that the effect of LIMK1 is accomplished through protein-protein interaction, a phenomenon that is sensitive to the stoichiometric balance and can be altered with a partial reduction in LIMK1 concentration. The putative role of LIMK1 as the regulator of both $\mathrm{G}_{2} / \mathrm{M}$ checkpoint and the invasive property of prostate cancer cells and a chromosomal gain on $7 \mathrm{q} 11.2$, the region of localization of LIMK1 gene, in metastatic prostate cancer suggest the possibility of LIMK1 as a cellular oncogene. Our study on identification of LIMK1 as a potential promoter of prostate cancer progression should facilitate further characterization of interacting partners involved in progression of primary tumors and metastasis of advanced tumors and might provide a novel therapeutic target for prevention of prostate cancer metastasis.

Acknowledgments-We thank C. Fernandez-Valle for extensive discussion and data analysis, L. Robles and C. Spadafora for help during the experiments, and J. Biggerstaff for help in confocal images.

\section{REFERENCES}

1. Okano, I., Hiraoka, J., Otera, H., Nunoue, K., Ohashi, K., Iwashita, S., Hirai, M. and Mizuno, K. (1995) J. Biol. Chem. 270, 31321-31330

2. Bernard, D., Geniatsus, S., Kannourakis, G., and Dringen, R. (1994) Cell Growth Differ. 5, 1159-1171

3. Wouters, C. H., Meijers-Heijboer, H. J., Eussen, B. J., van der Heide, A. A., van Luijk, R. B., van Drunen, E., Beverloo, B. B., Visscher, F., and Van Hemel,
J. O. (2001) Am. J. Med. Genet. 102, 261-265

4. Bamburg, J. R. (1999) Annu. Rev. Cell Dev. Biol. 15, 185-230

5. Arber, S., Barbayannis, F. A., Hanser, H., Schneider, C., Stanyon, C. A. Bernard, O., and Caroni, P. (1998) Nature 393, 805-809

6. Hall, A. (1998) Science 279, 509-514

7. Yang, N., Higuchi, O., Ohashi, K., Nagata, K., Wada, A., Kangawa, K., Nishida, E., and Mizuno, K. (1998) Nature 393, 809-812

8. Sumi, T., Matsumoto, K., Takai, Y., and Nakamura, T. (1999) J. Cell Biol. 147, 1519-1532

9. Edwards, D. C., Sanders, L. C., Bokoch, G. M., and Gill, G. N. (1999) Nat. Cell Biol. 1, 253-259

10. Ohashi, K., Nagata, K., Maekawa, M., Ishizaki, T., Narumiya, S., and Mizuno, K. (2000) J. Biol. Chem. 275, 3577-3582

11. Dan, C., Kelly, A., Bernard, O., and Minden, A. (2001) J. Biol. Chem. 276, $32115-32121$

12. Sumi, T., Matsumoto, K., Shibuya, A., and Nakamura, T. (2001) J. Biol. Chem. 276, 23092-23096

13. Yang, N., Higuchi, O., and Mizuno, K. (1998) Exp. Cell Res. 241, 242-252

14. Higuchi, O., Amano, T., Yang, N., and Mizuno, K. (1997) Oncogene 14, 1819-1825

15. Wang, J. Y., Frenzel, K. E., Wen, D., and Falls, D. (1998) J. Biol. Chem. 273, 20525-20534

16. Edwards, D., and Gill, G. N. (1999) J. Biol. Chem. 274, 11352-11361

17. Nobes, C. D., and Hall, A. (1995) Cell 81, 53-62

18. Small, J. V., Rottner, K., and Kaverina, I. (1999) Curr. Opin. Cell Biol. 11, $54-60$

19. Somlyo, A. V., Bradshaw, D., Ramos, S., Murphy, C., Myers, C. E., and Somlyo, A. P. (2000) Biochem. Biophys. Res. Commun. 269, 652-659

20. Yu, D., Wang, S., Dulski, K. M., Tsai, C. M., Nicolson, G. L., and Hung, M. C. (1994) Cancer Res. 54, 3260-3266

21. Morote, J., Torres, I, Caceres, C., Vallejo, C., Schwartz, S., and Reventos, J. (1999) Int. J. Cancer 84, 421-425

22. Dionne, C. A., Camoratto, A. M., Jani, J. P. Emerson, E., Neff, N., Vaught, J. L., Murakata, C., Djakiew, D., Lamb, J., Bova, S., George, D., and Issacs, J. T. (1998) Clin. Cancer Res, 4, 1887-1898

23. Walch, E. T., and Marchetti, D. (1999) Clin. Exp. Metastasis 17, 307-314

24. Malliri, A, Symons, M, Hennigan, R. F., Hurlstone, A. F., Lamb, R. F. Wheeler, T., and Ozanne, B. W. (1998) J. Cell Biol. 143, 1087-1099

25. Dhanasekaran, S. M., Barrette, T. R., Ghosh, D., Shah, R., Varambally, S. Kurachi, K., Pienta, K. J., Rubin, M. A., and Chinnaiyan, A. M. (2001) Nature 412, 822-826

26. Chakrabarti, R., Robles, L. D. Gibson, J., and Muroski, M. (2002) Cancer Genet. Cytogenet. 139, 115-125

27. Robles, L. D., Frost, A. R., Davila, M., Hutson, A. D., Grizzle, W. E., and Chakrabarti, R. (2002) J. Biol. Chem. 277, 25431-25438

28. Ellis, J. G., Davila, M., and Chakrabarti, R. (2003) J. Biol. Chem. 278, 1936-1945

29. Sumi, T., Matsumoto, K., and Nakamura, T. (2002) Biochem. Biophys. Res. Commun. 290, 1315-1320

30. Toshima, J., Toshima, J. Y., Takeuchi, K., Mori, R., and Mizuno, K. (2001) J. Biol. Chem. 276, 31449-31458

31. Higuchi, O., Baeg, G., Akiyama, T., and Mizuno, K. (1996) FEBS Lett. 396, 81-86

32. Amano, T., Kaji, N., Ohashi, K., and Mizuno, K. (2002) J. Biol. Chem. 277, 22093-22102

33. Strefford, J. C., Lillington, D. M., Young, B. D., and Oliver, R. T. (2001) Cancer Genet. Cytogenet. 124, 112-121

34. Defilippi, P., Olivo, C., Venturino, M., Dolce, L., Silengo, L., and Tarone, G. (1999) Microsc. Res. Tech. 47, 67-78

35. Turner, C. E. (2000) Nat. Cell Biol. 2, 231-236

36. Norman, J. C., Jones, D., Barry, S. T., Holt, M. R., Cockcroft, S., and Critchley, D. R. (1998) J. Cell Biol. 143, 1981-1995

37. Mizuno, K., Okano, I., Ohashi, K., Nunoue, K., Kuma, K., Miyata, T., and Nakamura, T. (1994) Oncogene 6, 1605-1612

38. Nagakawa, O., Murakami, K., Yamaura, T., Fujiuchi, Y., Murata, J., Fuse, H., and Saiki, I. (2000) Cancer Lett. 155, 173-179

39. Upadhyay, J., Shekarriz, B., Nemeth, J. A., Dong, Z., Cummings, G. D. Fridman, R., Sakr, W., Grignon, D. J., and Cher, M. L. (1999) Clin. Cancer Res. 5, 4105-4110

40. Itoh, Y., Takamura, A., Ito, N., Maru, Y., Sato, H., Suenaga, N., Aoki, T., and Seiki, M. (2001) EMBO J. 20, 4782-4793

41. Hotary, K., Allen, E., Punturieri, A., Yana, I., and Weiss, S. J. (2000) J. Cell Biol. 149, 1309-1323

42. Seiki, M. (1999) APMIS 107, 137-143

43. Alers, J. C., Rochat, J., Krijtenburg, P. J., Hop, W. C., Kranse, R., Rosenberg, C., Tanke, H. J., Schroder, F. H., and van Dekken, H. (2000) Lab. Invest. 80, 931-942

44. Alers, J. C., Krijtenburg, P. J., Hop, W. C., Bolle, W. A., Schroder, F. H., van der Kwast, T. H., Bosman, F. T., and van Dekken, H. (1998) J. Pathol. 185, 273-283

45. Alers, J. C., Krijtenburg, P. J., Vis, A. N., Hoedemaeker, R. F., Wildhagen M. F., Hop, W. C., van Der Kwast, T. T., Schroder, F. H., Tanke, H. J., and van Dekken, H. (2001) Am. J. Pathol. 158, 399-406

46. Alers, J. C., Krijtenburg, P. J., Rosenberg, C., Hop, W. C. J., Verkerk, A. M Schroder, F. H., van der Kwast, Th. H., Bosman, F. T., and van Dekken, H. (1997) Lab. Invest. 77, 437-448

47. Lian, J. P., Marks, P. G., Wang, J. Y., Falls, D. L., and Badwey, J. A. (2000) J. Biol. Chem. 275, 2869-2876 


\section{LIM Kinase 1 Is Essential for the Invasive Growth of Prostate Epithelial Cells:}

IMPLICATIONS IN PROSTATE CANCER

Monica Davila, Andra R. Frost, William E. Grizzle and Ratna Chakrabarti

J. Biol. Chem. 2003, 278:36868-36875.

doi: 10.1074/jbc.M306196200 originally published online June 23, 2003

Access the most updated version of this article at doi: 10.1074/jbc.M306196200

Alerts:

- When this article is cited

- When a correction for this article is posted

Click here to choose from all of JBC's e-mail alerts

This article cites 47 references, 21 of which can be accessed free at http://www.jbc.org/content/278/38/36868.full.html\#ref-list-1 